\title{
Full organic distributed feedback cavities based on a soluble electroluminescent oligothiophene
}

\author{
Dario Pisignano,* Elisa Mele, Luana Persano, Giuseppe Gigli, Paolo Visconti, and Roberto Cingolani \\ NNL, National Nanotechnology Laboratory of Istituto Nazionale di Fisica della Materia (INFM), c/o Dipartimento di Ingegneria \\ dell'Innovazione, via Arnesano, I-73100 Lecce, Italy \\ Giovanna Barbarella and Laura Favaretto \\ Consiglio Nazionale delle Ricerche (CNR), ISOF, Area della Ricerca di Bologna, via Gobetti 101, I-40129 Bologna, Italy
}

(Received 9 December 2003; revised manuscript received 20 July 2004; published 9 November 2004)

\begin{abstract}
We have studied the emission spectra of spin-coated films of a novel soluble substituted quinque-thiophene under nanoseconds pulsed excitation. The photoluminescence spectra show a clear line narrowing, ascribed to amplified spontaneous emission assisted by waveguiding inside the film slab, for excitation energy fluence above $0.8 \mathrm{~mJ} \mathrm{~cm}^{-2}$. We employed our oligothiophene as active medium for a fully organic distributed feedback (DFB) resonator on a flexible transparent substrate of poly(dimethylsiloxane) (PDMS). The DFB has been fabricated by replica molding from a master grating realized by electron-beam lithography, and subsequent spin coating of the active material on the patterned side of PDMS. It shows a clear DFB emission at about $603 \mathrm{~nm}$ of linewidth $0.8 \mathrm{~nm}$.
\end{abstract}

DOI: 10.1103/PhysRevB.70.205206

PACS number(s): 78.66.Qn

\section{INTRODUCTION}

Organic materials offer several appealing technological characteristics for the realization of photonic devices, the most important being the easy deposition on various substrates by evaporation, or spin coating in the case of soluble compounds, and the easy patterning by one-step soft lithographic techniques. ${ }^{1}$ In the last decade, the discovery of amplified emission from a number of organic compounds, both in solutions ${ }^{2}$ and in thin films, ${ }^{3}$ has driven the interest into organic semiconductor lasers, and into the photophysics of the excited state of molecules. So far, optical gain has been achieved in a number of conjugated compounds, ${ }^{4-12}$ and optically pumped laser action has been demonstrated for several cavity geometries. ${ }^{13-20}$

In this frame, functionalized thiophene-based oligomers have been demonstrated to be appealing candidates for photonics applications, because of their wide color tunability ${ }^{21}$ (which is connected to their structural properties, molecular conformation, and functionalization pattern); ${ }^{22}$ good chemical stability; ${ }^{23}$ high solubility in common organic solvents and very good film-forming properties. In the last years, the main drawbacks of unsubstituted oligothiophenes, namely, the low photoluminescence (PL) efficiency in the solid phase $\eta_{\mathrm{PL}}$, and the low-electron affinity $\chi$ have been overcome by the dioxide functionalization of the thiophene heteroatom, which allowed us to achieve values of $\eta_{\mathrm{PL}}$ up to $70 \%$ and of $\chi$ of about $3 \mathrm{~V}^{21}$ These features have been exploited to fabricate multicolor organic light-emitting diodes (OLEDs), with low turn-on voltage and high electroluminescence (EL) efficiency. $^{24,25}$

More recently tunable optical gain, not competing with photoinduced absorption, amplified spontaneous emission $(\mathrm{ASE})^{26}$ and single-mode lasing ${ }^{27}$ have been demonstrated in spin-coated films of different electroluminescent thienyl$S, S$-dioxide oligothiophenes. A crucial aspect of such results is that this class of materials allows one to obtain emission from blue to near infrared by exploiting the same chemistry, which enormously simplifies the synthesis and the fabrication of the device.

In this paper, we demonstrate optical gain under nanoseconds pulsed excitation in spin-cast films of the substituted thiophene-based pentamer, 3, 3', $4^{\prime \prime \prime}, 3^{\prime \prime \prime \prime}$-tetracyclohexyl$3^{\prime \prime}, 4^{\prime \prime}$-dihexyl-2,2' $: 5^{\prime}, 2^{\prime \prime}: 5^{\prime \prime}, 2^{\prime \prime \prime}: 5^{\prime \prime \prime}, 2^{\prime \prime \prime \prime}:$ quinquethiophene$1^{\prime \prime}, 1^{\prime \prime}$-dioxide T5oCx, M.W.=940 g/mol, structure in the inset of Fig. 1). This molecule has been previously used to fabricate highly efficient lasers ${ }^{28}$ and OLEDs, exhibiting luminance of $400 \mathrm{~cd} / \mathrm{m}^{2}$ (at $20 \mathrm{~V}$ ) and EL efficiency of $0.2 \%$ for several hours in air atmosphere. ${ }^{25}$ We observed line narrowing ascribed to ASE, assisted by the waveguiding effect due to the different refractive indexes $n$ of the substrate, organic film, and air, respectively. The determined threshold excitation density for stimulated emission amounts to $2.6 \mathrm{~mJ} \mathrm{~cm}^{-2}$, whereas the spectral dependence of the net optical gain, revealing a two-peaks response to photoexcitation, shows an absolute maximum of $7.0 \mathrm{~cm}^{-1}$ at $625 \mathrm{~nm}$. Such

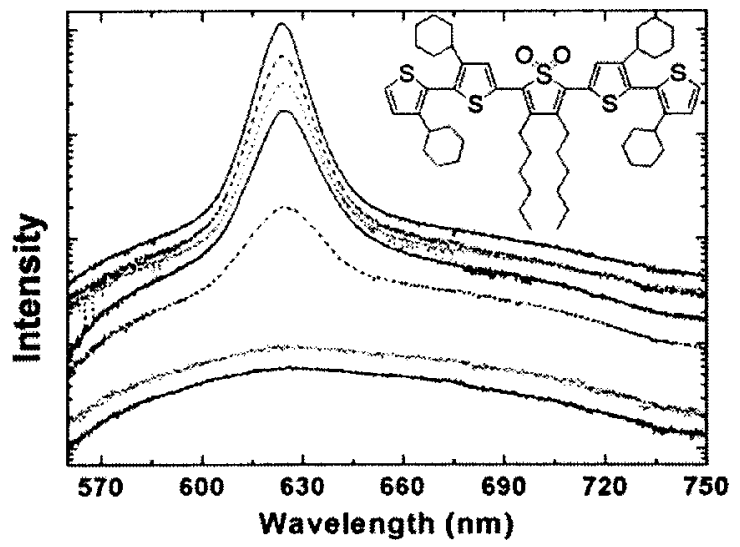

FIG. 1. Emission spectra from T5oCx films under different absorbed excitation fluences (from bottom to top: 0.2, 0.6, 1.6, 1.7,1.9, $2.4,6.1 \mathrm{~mJ} \mathrm{~cm}^{-2}$ ). Inset: molecular structure. T5oCx was synthesized according to the modalities given in Ref. 50 . 
gain properties have been exploited to realize a fully organic distributed feedback (DFB) cavity made by soft lithography on a flexible transparent substrate. The device exhibits single-mode emission around $603 \mathrm{~nm}$ of linewidth less than $1 \mathrm{~nm}$.

\section{EXPERIMENT}

T5oCx films were cast by spin coating on Corning glass substrates $(n=1.46)$. With a spin-coating speed of $1600 \mathrm{rpm}$ we obtained neat films of thickness about $440 \mathrm{~nm}$, measured by a profilometer. To fabricate the DFB structures by soft lithography, we first realized a $\mathrm{Si}$ master grating of period

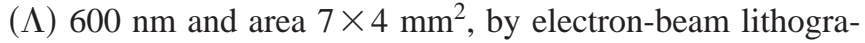
phy using a Leica LION LVI system working at an acceleration energy of $2.5 \mathrm{keV}$ and subsequent reactive ion etching by a CF-Ar mixture chosen to minimize the plasma-induced polymer deposition. The pattern was then transferred into poly(dimethylsiloxane) (PDMS) by polymerization according to standard replica molding procedures. ${ }^{1}$ The original master was then replicated onto an elastomeric negative copy, on which we directly spin-coated $\mathrm{T} 5 \mathrm{oCx}$ as active medium of the DFB resonator.

The third harmonic $(\lambda=355 \mathrm{~nm})$ of $3 \mathrm{~ns} Q$-switched Nd:YAG (YAG-yttrium aluminum garnet) laser (SpectraPhysics, repetition rate of $10 \mathrm{~Hz}$ ) was employed as the excitation source for both the spin-coated films and the DFB. The excitation beam was focused on the sample by a cylindrical lens, thus providing a rectangular excitation stripe of width $500 \mu \mathrm{m}$. The sample emission at room temperature was collected from the sample edge, dispersed by a monochromator, and detected by a Si charge coupled device. The transmission spectra of the elastomeric elements before and after spin casting the active medium were measured by a Varian Cary 5000 spectrophotometer. The optical measurements were carried out under a continuous $\mathrm{N}_{2}$ flux or under vacuum $\left(10^{-6} \mathrm{mbar}\right)$ to inhibit photo-oxidation.

\section{RESULTS AND DISCUSSION}

The emission spectra of the $\mathrm{T} 5 \mathrm{oCx}$ films under nanosecond excitation pulses of different power are shown in Fig. 1. At low absorbed excitation powers (less than about $1 \mathrm{~mJ} / \mathrm{cm}^{-2}$ ), only a broad full width at half maximum (FWHM) $>120 \mathrm{~nm}$ and featureless PL spectrum is visible, whereas, for higher pump energies, a narrow peak, ascribed to ASE assisted by a waveguiding effect due to the different refractive index of substrate, gain medium, and air, appears at $625 \mathrm{~nm}$. The broad PL emission and the narrow peak exhibit different dependences on the excitation intensity, as shown in Fig. 2. The PL signal weakly grows in the investigated intensity range (empty dots in Fig. 2), whereas the total intensity $I$, due to the narrow band, shows an abrupt (approximately exponential) increase up to about $1.9 \mathrm{~mJ} / \mathrm{cm}^{-2}$, without distinct threshold, and then saturates for very large excitation intensities $\left(E>3 \mathrm{~mJ} / \mathrm{cm}^{-2}\right)$. Such a sublinear evolution of the integrated intensity indicates the presence of non-radiative decay processes, increasing with increasing the pump density. The resulting gain saturation has been mainly

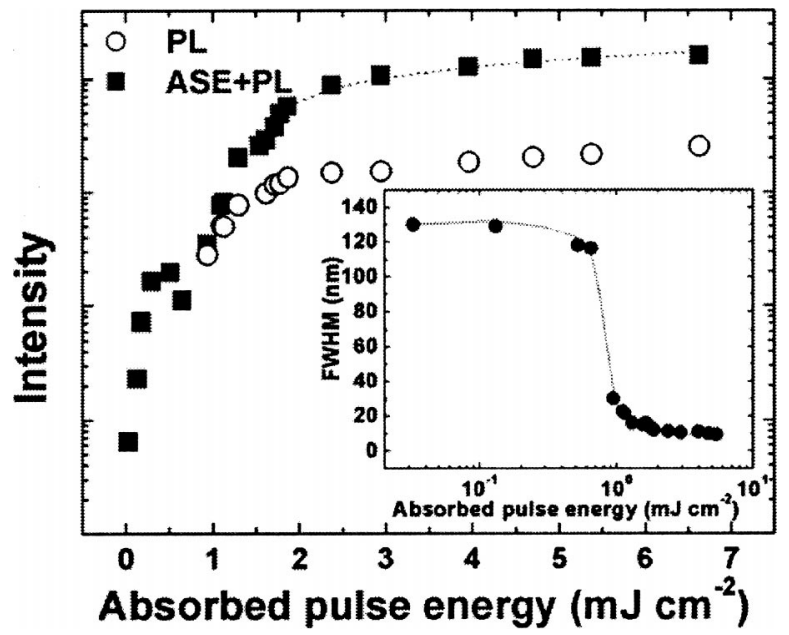

FIG. 2. Dependence of the total integrated intensity (ASE $+\mathrm{PL})$ and of the PL on the excitation energy fluence. The dotted line is the best fit of the data between 2.4 and $6.6 \mathrm{~mJ} \mathrm{~cm}^{-2}$ to the square root behavior predicted by the bimolecular annihilation model. Inset: emission linewidth (FWHM) as a function of pump pulse energy density. The continuous line is a guide for the eye.

attributed to nonlinear effects, such as bimolecular exciton annihilation, induced by the high density of excitons formed under very intense photon fluxes. ${ }^{29}$ Such a behavior is confirmed by the square root dependence of the high power emission on the excitation density, which is consistent with the bimolecular annihilation model (dotted curve fitting the data in Fig. 2). 6,29

The narrowing of the spectral linewidth occurs for pump densities above $\approx 0.8 \mathrm{~mJ} / \mathrm{cm}^{-2}$, and reaches a best value of FWHM $\approx 9 \mathrm{~nm}$ at $6.1 \mathrm{~mJ} / \mathrm{cm}^{-2}$ (inset of Fig. 2). We remind that previously reported line narrowing thresholds for polymers under nanoseconds excitation reached values as high as $14 \mathrm{~mJ} \mathrm{~cm}^{-2}$ for $m$-LPPP. ${ }^{10}$ Moreover, since line narrowing stops when gain saturation occurs, these findings demonstrate that high pump fluences can be applied to T5oCx films without preventing a further increase of the emitted intensity and a reduction of the spectral broadening, thus making this molecule very appealing for laser applications.

A quantitative assessment of the gain properties of $\mathrm{T} 5 \mathrm{oCx}$ was obtained by the variable stripe length method, providing the optical gain spectrum $g(\lambda)$ of $\mathrm{T} 5 \mathrm{oCx}$ via the relation between the emitted intensity $I$, and the excitation stripe length $l:{ }^{30}$

$$
I(\lambda)=I_{0}(\lambda)\{\exp [g(\lambda) l]-1\} .
$$

Interestingly, the gain spectrum shows two peaks (Fig. 3): a high-energy peak, centered at $625 \mathrm{~nm}$ with a maximum of $7.0 \mathrm{~cm}^{-1}$, and a low-energy one, not visible in the emission spectra because of the corresponding low value of $I_{0}(\lambda)$, centered around $740 \mathrm{~nm}$, with a maximum of $3.5 \mathrm{~cm}^{-1}$. These gain coefficients are comparable to those reported for other organic optical amplifiers, such as thin films of infrared dyedoped poly(methylmethacrylate), ${ }^{31}$ although they are still one order of magnitude lower than the best values reported for conjugated polymers, such as the poly(2-butyl-5-( $\left(2^{\prime}-\right.$ 


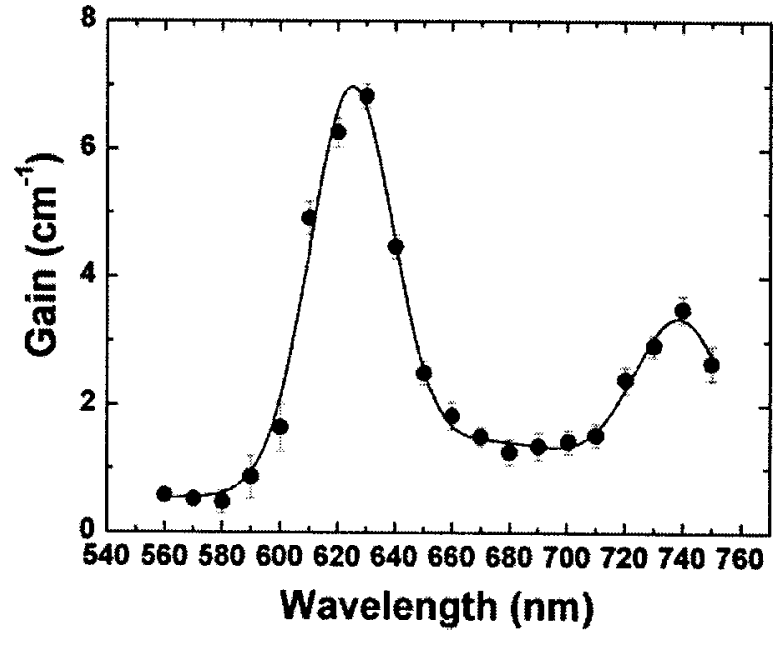

FIG. 3. Optical gain spectrum for an absorbed excitation density of $960 \mu \mathrm{J} \mathrm{cm}^{-2}$. The continuous line is a guide for the eye.

ethylhexyl)-1,4-phenylene -vinylene, the 2,5-dioctyloxy poly ( $p$-phenylene-vinylene $),{ }^{5}$ and some polyfluorenes. ${ }^{12}$

The two-peaks gain spectrum here observed is consistent with the occurrence of ASE. In fact, as the maxima of the net gain are due both to intrinsic amplified emission of the material and to waveguiding effect, optical amplification can be observed at different wavelengths. Multipeaks ASE lines have been previously reported for oligothiophene single crystals, the dominating emission being attributed to the spectral selection due either to sample thickness or to structural defects. ${ }^{32}$

The $\mathrm{T} 5 \mathrm{oCx}$ has been demonstrated to have a $S_{1}$-dominated energy excitation scheme particularly suitable for optoelectronic applications. ${ }^{33}$ The substituents groups mainly act as barrier for the strong coupling between close molecules, often occurring in linear oligothiophenes, thus reducing the intermolecular nonradiative recombination in the solid state, and also contributing to strongly localize the lowest unoccupied molecular orbital on the central thiophene ring. ${ }^{33}$ Finally, they also determine the excellent filmforming properties of this material by the high disorder induced within the organic slab, limiting the formation of crystalline domains in the solid state.

In particular, the occurrence of optical gain under longpulse (ns) pump conditions and the strong chemical stability suggest that $\mathrm{T} 5 \mathrm{oCx}$ is an excellent active medium for organic lasers operating under steady state excitation and under high injection densities. In this context, DFB resonators offer unsurpassed wavelength selectivity with respect to conventional cavities, however their spectral characteristics can be negatively affected by the slight distortions induced by fabrication techniques on the organic materials, which may cause broad or multimode laser emission. The distributed feedback inside a DFB resonator ${ }^{34}$ is due to the spatial modulation of the refractive index $n$ or of the gain of the propagation medium, resulting in a DFB emission which can be double mode or single mode, respectively. The lasing wavelength $\lambda_{m}$ is related to the cavity geometry by the relation
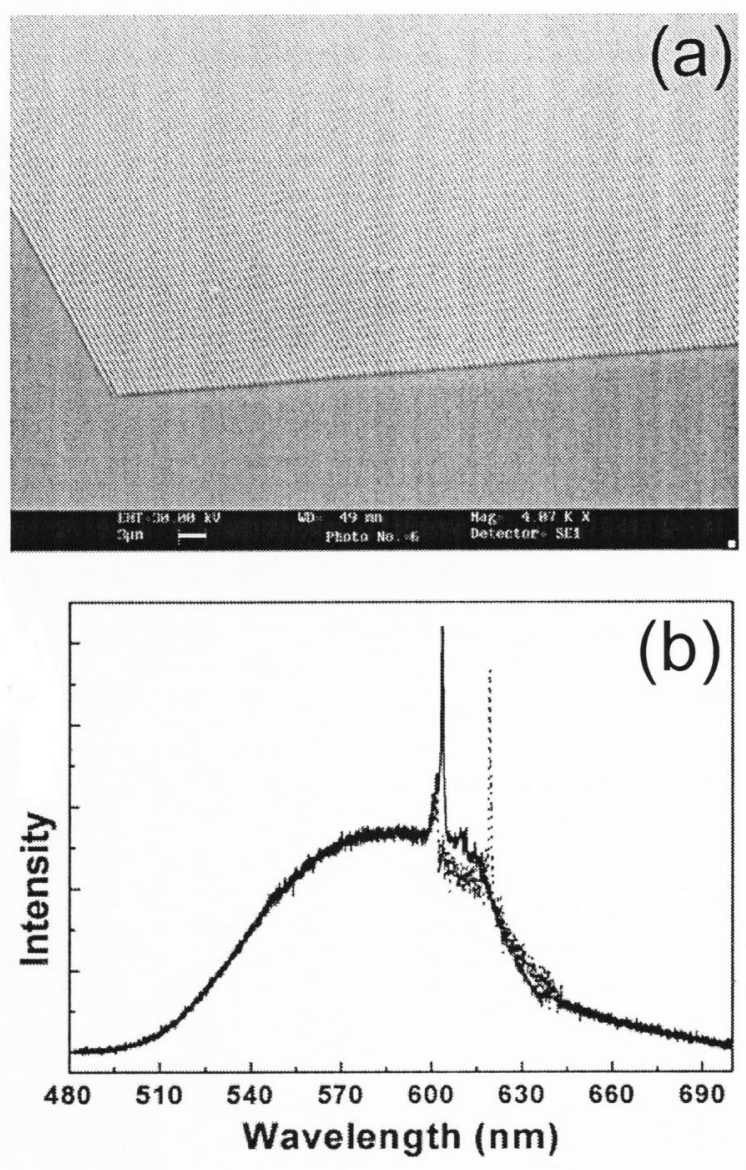

FIG. 4. (a) Master Si DFB structure by SEM; (b) spectra of the PDMS/T5oCx DFB device (at an absorbed photoexcitation density of $13.6 \mu \mathrm{J}$ pulse, corresponding to about $125 \mathrm{~kW} / \mathrm{cm}^{2}$ ). Modes emitted from different sample regions are peaked at 603 (solid line) and $619 \mathrm{~nm}$ (dotted line). A less intense mode is also visible at $602 \mathrm{~nm}$ in the regions lasing at $619 \mathrm{~nm}$ (dotted line).

$$
m \lambda_{m}=2 n_{\mathrm{eff}} \Lambda
$$

where $m$ is the diffraction order of the DFB grating and $n_{\text {eff }}$ indicates the effective refractive index of the propagating mode.

Our master DFB structure, imaged by scanning electron microscopy (SEM), is shown in Fig. 4(a). As the T5oCx layer in the device is sandwiched between air and PDMS $(n=1.41),{ }^{35}$ the directionality of the DFB edge emission is favored by the waveguiding effect of the film slab. As the PDMS corrugated surface directly interfaces with the active medium, both $n$ and the gain are spatially modulated in our case. In order to test the uniformity of the PDMS gratings in the direction parallel to the grooves, we collected the DFB spectrum by photopumping different regions within the patterned area. Typical spectra of our DFB device are shown in Fig. 4(b). A single-mode emission, with a linewidth as narrow as $0.8 \mathrm{~nm}$, is clearly observed at $603 \mathrm{~nm}$ (solid line) superimposed to the broad spontaneous background. The observed predominant single-mode emission suggests that the feedback mechanisms is dominated by the periodic variation 


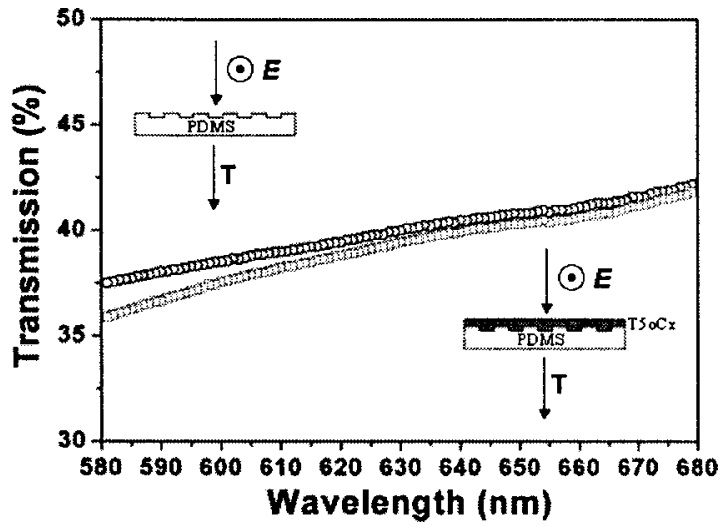

FIG. 5. Plot of the transmission (T) of $s$-polarized light (electric field $\mathbf{E}$, parallel to the grating grooves) before (circles, top inset) and after (squares, bottom inset) covering the elastomeric periodic nanostructure by the oligothiophene-S,S-dioxide.

of the optical gain along the slab. In a few regions, another narrow peak was detected at $619 \mathrm{~nm}$ (FWHM=0.6 nm, dotted line). No multimode emission was observed, indicating the high degree of uniformity of the fabricated nanostructure. According to Eq. (2), this device shows DFB emission corresponding to the third order of the grating, with an effective refractive index around 1.5.

The Bragg diffraction induced by the periodic corrugation, continuously reflecting a part of the wave propagating in the forward direction of the medium, is responsible for the lasing action. The DFB emission, namely, the coupling of the bound optical modes with the free radiation, is usually closely related to the band structure of the one-dimensional photonic crystal structure, occurring mostly at the band edge corresponding to the lowest cavity losses. However, in our case we could not appreciate a strong fluorescence dip evidencing a redistribution of the optical density of states as a consequence of the one-dimensional periodic nanostructure. This has been often found in organic DFBs, for instance, employing nonmorphological ${ }^{36,37}$ or photoinduced surface-periodic ${ }^{38,39}$ gratings, and in our case is likely related to the weak feedback along the organic slab, suggested by the presence of the considerable spontaneous fluorescence signal [Fig. 4(b)].

The weakness of the feedback provided by the grating is also indicated by the normal transmission spectra of the device (Fig. 5). The transmission spectrum is not significantly affected by the absorption of PDMS and T5oCx, whose long-wavelength edge is at around $550 \mathrm{~nm}$. The transmitted component is therefore reduced mainly by the amount of light guided in the high refractive index organic slab. In addition, both for the uncovered elastomeric grating and for the complete PDMS-T5oCx DFB, we observed very smooth transmission characteristics for $s$-polarized light (polarized parallel to the grating grooves, namely, corresponding to transversal electric guided modes). In particular, accordingly to what observed for fluorescence, the transmission spectra do not exhibit dip in the region of the lasing wavelengths which would evidence an enhanced coupling of light, and which would be the signature of the relation between the photon band structure and the emission wavelength of organic DFBs similarly to findings for polymer-based grating resonators. ${ }^{40}$ Similar characteristics were found for light polarized perpendicularly to the grating grooves. We believe that this is mainly due to the poor refractive index contrast along the nanostructure. This likely enhances the importance of the power-dependent optical gain modulation, mainly responsible for the $603 \mathrm{~nm}$ lasing action at high excitation densities. $^{41}$

The overall feedback efficiency likely increases in the regions where we observed the long-wavelength $(619 \mathrm{~nm})$ peak, which is indeed relatively more intense and narrower than the peak at $603 \mathrm{~nm}$. The cavity quality factor $Q$ $=\Delta \lambda / \lambda$ of the distributed feedback microcavity in the two regions is 1030 and 750, respectively. This indicates a higher finesse of the long-wavelength coupled mode, which can be related to a weaker output coupling, namely, to lower cavity losses. Correspondingly, in the device regions of higher feedback we see the occurrence of a photon gap between the two lasing peaks at 602 and $619 \mathrm{~nm}$ [dotted line in Fig. 4(b)].

To date, organic DFB have been realized by conventional ${ }^{15,40-42}$ and soft lithographic methods, ${ }^{17,43-47}$ and by exploiting as active medium conjugated polymers, ${ }^{15,40-43}$ and blends incorporating commercial dyes. ${ }^{17,36-38,44-47}$ Among other soft lithography techniques, the direct spin coating of the active medium after replica molding ${ }^{17}$ here employed offers the simplest fabrication route, namely, the highest degree of uniformity and the lowest number of defects in the nanostructure nearby the optically active layer. These results confirm that soft lithography can be employed to realize phase-sensitive optical devices. ${ }^{48}$ Moreover, several different applications can be envisaged for elastomerbased active optical devices, including tunability due to the easy deformation of the optical surfaces, as demonstrated by the fabrication of elastomeric passive optical components. ${ }^{49}$

\section{CONCLUSION}

In conclusion, in the present work we employed the electroluminescent thiophene-based oligomer, T5oCx, as the active medium for a high-quality full-organic DFB resonator fabricated by soft lithography. By directly spin coating the active layer onto a patterned elastomeric surface, we observed a clear DFB emission. In addition, the line narrowing due to ASE and the spectral dependence of optical gain from spin coated films of $\mathrm{T} 5 \mathrm{oCx}$ were investigated by means of photopumped emission under nanosecond-pulsed excitation. The high pump threshold for saturation, together with the excellent processability and film-forming capability, clearly indicate that thienyl-S,S-dioxide oligomers are appealing competitors of conjugated polymers for the realization of organic-based semiconductor lasers. 
*Also at Dipartimento di Fisca, Università di Lecce, via Amesano, I-73100 Lecce, Italy. Electronic mail: dario.pisignano@unile.it

${ }^{1}$ Y. Xia and G. M. Whitesides, Angew. Chem., Int. Ed. 37, 550 (1998).

${ }^{2}$ D. Moses, Appl. Phys. Lett. 60, 3215 (1992).

${ }^{3}$ N. Tessler, G. J. Denton, and R. H. Friend, Nature (London) 382, 695 (1996).

${ }^{4}$ W. Graupner, G. Leising, G. Lanzani, M. Nisoli, S. De Silvestri, and U. Scherf, Phys. Rev. Lett. 76, 847 (1996).

${ }^{5}$ S. V. Frolov, W. Gellerman, M. Ozaki, K. Yoshino, and Z. V. Vardeny, Phys. Rev. Lett. 78, 729 (1997).

${ }^{6}$ G. J. Denton, N. Tessler, M. A. Stevens, and R. H. Friend, Adv. Mater. (Weinheim, Ger.) 9, 547 (1997).

${ }^{7}$ G. H. Gelink, J. W. Warman, M. Remmers, and D. Neher, Chem. Phys. Lett. 265, 320 (1997).

${ }^{8}$ S. Stagira, M. Nisoli, G. Cerullo, M. Zavelani-Rossi, S. De Silvestri, G. Lanzani, W. Graupner, and G. Leising, Chem. Phys. Lett. 289, 205 (1998).

${ }^{9}$ B. Schweitzer, G. Wengmann, H. Giessen, D. Hertel, H. Bassler, and R. F. Mahrt, Appl. Phys. Lett. 72, 2933 (1998).

${ }^{10}$ H. J. Brouwer, V. V. Krasnikov, T. A. Pham, R. E. Gill, and G. Hadziioannou, Appl. Phys. Lett. 73, 708 (1998).

${ }^{11}$ C. Spiegelberg, N. Peyhambarian, and B. Kippelen, Appl. Phys. Lett. 75, 748 (1999).

${ }^{12}$ R. Xia, G. Heliotis, and D. D. C. Bradley, Appl. Phys. Lett. 82, 3599 (2003).

${ }^{13}$ S. V. Frolov, M. Shkunov, Z. V. Vardeny, and K. Yoshino, Phys. Rev. B 56, R4363 (1997).

${ }^{14}$ V. G. Kozlov, V. Bulović, P. E. Burrows, and S. R. Forrest, Nature (London) 389, 362 (1997).

${ }^{15}$ M. Bergreen, A. Dodabalapur, R. E. Slusher, and Z. Bao, Nature (London) 389, 466 (1997).

${ }^{16}$ M. Bergreen, A. Dodabalapur, and R. E. Slusher, Appl. Phys. Lett. 71, 2230 (1997).

${ }^{17}$ M. Bergreen, A. Dodabalapur, R. E. Slusher, A. Timko, and O. Nalamasu, Appl. Phys. Lett. 72, 410 (1998).

${ }^{18}$ S. Stagira, M. Zavelani-Rossi, M. Nisoli, S. De Silvestri, G. Lanzani, C. Zenz, P. Mataloni, and G. Leising., Appl. Phys. Lett. 73, 2860 (1998).

${ }^{19}$ M. D. McGehee, M. A. Diaz-Garcia, F. Hide, R. Gupta, E. K. Miller, D. Moses, and A. J. Heeger, Appl. Phys. Lett. 72, 1536 (1998).

${ }^{20}$ C. Kallinger, M. Hilmer, A. Haugeneder, M. Perner, W. Spirkl, U. Lemmer, J. Feldmann, U. Scherf, K. Mullen, A. Gombert, and V. Wittwer, Adv. Mater. (Weinheim, Ger.) 10, 920 (1998).

${ }^{21}$ M. Anni, G. Gigli, V. Paladini, R. Cingolani, G. Barbarella, L. Favaretto, G. Sotgiu, and M. Zambianchi, Appl. Phys. Lett. 77, 2458 (2000).

${ }^{22}$ G. Gigli, M. Lomascolo, R. Cingolani, G. Barbarella, M. Zambianchi, L. Antolini, F. Della Sala, A. Di Carlo, and P. Lugli, Appl. Phys. Lett. 73, 2414 (1998).

${ }^{23}$ G. Barbarella, L. Favaretto, M. Zambianchi, O. Pudova, C. Arbizzani, A. Bongini, and M. Mastragostino, Adv. Mater. (Weinheim, Ger.) 10, 551 (1998).

${ }^{24}$ G. Gigli, G. Barbarella, L. Favaretto, F. Cacialli, and R. Cingolani, Appl. Phys. Lett. 75, 439 (1999).

${ }^{25}$ G. Gigli, O. Inganas, M. Anni, M. De Vittorio, R. Cingolani, G. Barbarella, and L. Favaretto, Appl. Phys. Lett. 78, 1493 (2001).
${ }^{26}$ M. Anni, G. Gigli, R. Cingolani, M. Zavelani-Rossi, C. Gadermaier, G. Lanzani, G. Barbarella, and L. Favaretto, Appl. Phys. Lett. 78, 2679 (2001).

${ }^{27}$ M. Zavelani-Rossi, G. Lanzani, S. De Silvestri, M. Anni, G. Gigli, R. Cingolani, G. Barbarella, and L. Favaretto, Appl. Phys. Lett. 79, 4082 (2001).

${ }^{28}$ D. Pisignano, M. Anni, G. Gigli, R. Cingolani, M. ZavelaniRossi, G. Lanzani, G. Barbarella, and L. Favaretto, Appl. Phys. Lett. 81, 3534 (2002).

${ }^{29}$ E. S. Maniloff, V. I. Klimov, and D. W. McBranch, Phys. Rev. B 56, 1876 (1997).

${ }^{30}$ K. L. Shaklee, R. E. Nahory, and R. F. Leheny, J. Lumin. 7, 284 (1973).

${ }^{31}$ J. Thompson, M. Anni, S. Lattante, D. Pisignano, R. I. R. Blyth, G. Gigli, and R. Cingolani, Synth. Met. 143, 305 (2004).

${ }^{32}$ D. Fichou, V. Dumarcher, and J.-M. Nunzi, Synth. Met. 101, 610 (1999).

${ }^{33}$ D. Pisignano, F. Della Sala, L. Persano, G. Gigli, R. Cingolani, G. Barbarella, and L. Favaretto, Physica A 339, 106 (2004).

${ }^{34}$ H. Kogelnik and C. V. Shank, Appl. Phys. Lett. 18, 152 (1971).

${ }^{35}$ O. J. A. Schueller, X.-M. Zhao, G. M. Whitesides, S. P. Smith, and M. Prentiss, Adv. Mater. (Weinheim, Ger.) 11, 37 (1999).

${ }^{36}$ M. Nagawa, M. Ichikawa, T. Koyama, H. Shirai, Y. Taniguchi, A. Hongo, S. Tsuji, and Y. Nakano, Appl. Phys. Lett. 77, 2641 (2000).

${ }^{37}$ N. Tsutsumi, T. Kawahira, and W. Sakai, Appl. Phys. Lett. 83, 2533 (2003).

${ }^{38}$ V. Dumarcher, L. Rocha, C. Denis, C. Fiorini, J.-M. Nunzi, F. Sobel, B. Sahraoui, and D. Gindre, J. Opt. A, Pure Appl. Opt. 2, 279 (2000).

${ }^{39}$ T. Maillou, J. L. Moigne, V. Dumarcher, L. Rocha, B. Geffory, and J.-M. Nunzi, Adv. Mater. (Weinheim, Ger.) 14, 1297 (2002).

${ }^{40}$ G. A. Turnbull, P. Andrew, M. J. Jory, W. L. Barnes, and I. D. W. Samuel, Phys. Rev. B 64, 125122 (2001).

${ }^{41}$ G. Heliotis, R. Xia, G. A. Turnbull, P. Andrew, M. J. Jory, W. L. Barnes, I. D. W. Samuel, and D. D. C. Bradley, Adv. Funct. Mater. 14, 91 (2004).

${ }^{42}$ N. Moll, R. F. Mahrt, C. Bauer, H. Giessen, B. Schnabel, E. B. Kley, and U. Scherf, Appl. Phys. Lett. 80, 734 (2002).

${ }^{43}$ S. Riechel, C. Kallinger, U. Lemmer, J. Feldmann, A. Gombert, V. Wittwer, and U. Scherf, Appl. Phys. Lett. 77, 2310 (2000).

${ }^{44}$ J. A. Rogers, M. Meier, and A. Dodabalapur, Appl. Phys. Lett. 73, 1766 (1998).

${ }^{45}$ J. A. Rogers, M. Meier, A. Dodabalapur, E. J. Laskowski, and M. A. Cappuzzo, Appl. Phys. Lett. 74, 3257 (1999).

${ }^{46}$ B. J. Scott, G. Wirnsberger, M. D. Mc.Gehee, B. F. Chmelka, and G. D. Stucky, Adv. Mater. (Weinheim, Ger.) 13, 1231 (2001).

${ }^{47}$ S. Riechel, U. Lemmer, J. Feldmann, S. Berleb, A. G. Mückl, W. Brütting, A. Gombert, and V. Wittwer, Opt. Lett. 26, 593 (2001).

${ }^{48}$ D. Pisignano, G. Gigli, P. Visconti, A. Zocco, A. Perrone, and R. Cingolani, J. Vac. Sci. Technol. B 20, 2248 (2002).

${ }^{49}$ J. L. Wilbur, R. J. Jackman, G. M. Whitesides, E. L. Cheung, L. K. Lee, and M. G. Prentiss, Chem. Mater. 8, 1380 (1996).

${ }^{50}$ G. Barbarella, L. Favaretto, G. Sotgiu, M. Zambianchi, A. Bongini, C. Arbizzani, M. Mastragostino, M. Anni, G. Gigli, and R. Cingolani, J. Am. Chem. Soc. 122, 11971 (2000). 